\title{
Increased placental growth factor (PIGF) concentrations in children and adolescents with obesity and the metabolic syndrome
}

\author{
Panagiota Pervanidou, ${ }^{1}$ Giorgos Chouliaras, ${ }^{1}$ Athanassios Akalestos, ${ }^{2}$ \\ Despoina Bastaki, ${ }^{1}$ Filia Apostolakou, ${ }^{2}$ Ioannis Papassotiriou, ${ }^{2}$ George P. Chrousos ${ }^{1,3}$ \\ ${ }^{1}$ Childhood Obesity Clinic, First Department of Pediatrics, University of Athens Medical School, Aghia Sophia Children's \\ Hospital; ${ }^{2}$ Department of Clinical Biochemistry, Aghia Sophia Children's Hospital; Athens, Greece; ${ }^{3}$ Department of \\ Biochemistry, King Abdulaziz University, Jeddah, KSA
}

\begin{abstract}
OBJECTIVE: Childhood obesity and the Metabolic Syndrome (MetS) are associated with an increased risk for early onset endothelial dysfunction and atherosclerosis. Placental growth factor (PIGF), a member of the vascular endothelial growth factor family, plays an important role in atherosclerosis by stimulating angiogenesis and atherogenic migration of monocytes/ macrophages into the arterial wall. The aim of this study was to investigate differences in circulating PIGF concentrations between children with obesity/metabolic syndrome and non-obese children. We have previously shown increased high-sensitivity troponin (hs-TnT) concentrations in children with MetS from the same cohort. DESIGN: Fifty-seven obese (49 without and 8 with MetS) and 25 non-obese children (controls) were assessed at the Childhood Obesity Clinic of our Department. Obesity was defined using the IOTF criteria. MetS was defined based on the IDF criteria. PIGF was measured using electrochemiluminescence methodology. RESULTS: Mean PIGF concentrations of obese children were significantly higher $(p=0.048)$ compared with those of the controls. Analysis of the three groups, the obese (without MetS), the MetS and the control, demonstrated a significant difference in PIGF concentrations $(p=0.035)$. Subgroup analysis revealed increased PIGF concentrations in children with the MetS compared to the controls $(p=0.009)$. Troponin had a significant positive correlation with PIGF overall $(p=0.003)$ and in the obese group $(p=0.046)$. CONCLUSIONS: Increased serum concentrations of PIGF, a biomarker of angiogenesis, are found in obese children with the MetS compared to non-obese controls, whereas PIGF correlated positively with troponin. Longitudinal studies may reveal the prognostic role of this biomarker in the progression of atherosclerosis in obese children with the MetS.
\end{abstract}

Key words: Angiogenesis, Cardiovascular risk, Children, Metabolic syndrome, Obesity, PlGF, Troponin 


\section{INTRODUCTION}

Childhood obesity and related comorbidities, such as dyslipidemia, arterial hypertension and impaired glucose metabolism, collectively defined as Metabolic Syndrome (MetS), are associated with an increased risk for early onset endothelial dysfunction and atherosclerosis. ${ }^{1,2}$ Angiogenesis, the physiological formation of new blood vessels, is crucial for embryonic and postnatal growth, as well as for a variety of complex biological processes during adulthood, but also constitutes a major pathological mechanism associated with the progression of atherosclerosis. ${ }^{3,4}$ Most atherosclerotic regions are vascularized by a large network of small neo-vessels that play an important role in plaque formation and development. Angiogenesis is induced by hypoxia and inflammation in the atherosclerotic area and is mainly regulated by members of the vascular endothelial growth factor (VEGF) family, their receptors and placental growth factor (PIGF). ${ }^{4-6}$

Increased serum VEGF concentrations have been found in patients with unstable carotid artery lesions. ${ }^{\text {? }}$ It is also well known that the VEGF system accounts for most of the angiogenic activity in the adipose tissue, ${ }^{8}$ whereas several studies have revealed increased serum VEGF concentrations in obese adults and children. ${ }^{9-11}$ In addition, a recent study has shown that weight loss was associated with a reduced angiogenic activity in the circulation. ${ }^{12}$

PIGF is highly expressed in placenta throughout gestation, but it is also expressed at a low level in the heart, lung, skeletal muscle, adipose tissue and other organs. There is evidence that PIGF plays an important role in a range of physiological and pathological conditions including preeclampsia, cardiovascular disease (CVD) and tumor progression. ${ }^{5,6,13} \mathrm{PlGF}$, by stimulating angiogenesis and atherogenic migration of monocytes/macrophages into the arterial wall, promoted atherosclerotic intimal thickening in hypercholesterolemic rabbits and macrophage accumulation in early atherosclerotic lesions in apolipoprotein-E deficient mice. ${ }^{13}$ Furthermore, the development of obesity per se is associated with significant growth of adipose tissue, i.e. adipogenesis, which is tightly associated with angiogenesis. ${ }^{14}$ In murine models of diet-induced obesity, inactivation of PIGF impaired adipose tissue development, partly resulting from reduced angiogenesis. ${ }^{15}$

A very limited number of clinical data have shown increased PIGF concentrations in obese adults with the MetS compared to non-MetS individuals, after adjustment for age, smoking and body mass index (BMI). ${ }^{16}$ The same research team examined PIGF concentrations in obese children ${ }^{10}$ but did not find significant differences. In our study, we aimed to investigate circulating PlGF concentrations in children with obesity and/or MetS in comparison to non-obese children. We hypothesized higher PIGF concentrations in children with the MetS.

We previously demonstrated increased - although within the normal range - concentrations of high sensitivity troponin (hs-TnT) in the same cohort of children with MetS, ${ }^{17}$ compared to the obese group without the MetS and to the non-obese group. Our previous findings imply that subclinical atherosclerosis during childhood may progress more rapidly in obese children with the MetS and that the minimal increase of hs-TnT may be associated with subclinical cardiac damage. Since PIGF is a potential independent predictor of coronary heart disease $\mathrm{e}^{6}$ in adults, we decided also to examine associations between these two markers, hs-TnT and PIGF, in our cohort.

\section{PATIENTS AND METHODS}

We have previously described the study design and methods, ${ }^{17}$ which we summarize as follows: The study was approved by the Ethics Committee of "Aghia Sophia" Children's Hospital and written informed consent was obtained from the participants and their parents. We included children 7-13yrs old, assessed at the Obesity Clinic. Exclusion criteria were: chronic illnesses, chronic use of medication, syndromic obesity, intellectual or psychiatric disorders and chromosomal disorders.

Obesity was defined using the IOTF criteria. ${ }^{18}$ BMI z-scores were calculated based on the Greek growth charts..$^{19}$ MetS was defined based on the IDF criteria. ${ }^{20}$ Participants' pubertal development was determined by physical examination based on the 5 Tanner Stages. ${ }^{21}$ Children at stages (for genital development in boys and breast development in girls) 2-5 were characterized as pubertal. 
Three groups were formed: controls (CG), obese without MetS (ObMS-) and obese with MetS (ObMS+). The ObMS- and ObMS+ groups were also pooled to form the combined obesity group $(\mathrm{Ob})$. In addition to obesity, low HDL-C/high triglycerides defined MetS in 7 children, and high triglycerides/ high systolic blood pressure in 1 male child.

The blood samples were collected in the overnight fasting state during a scheduled visit at 8.00-9.00 a.m. The blood was centrifuged and the serum obtained was stored at $-85^{\circ} \mathrm{C}$. Serum glucose, HDL, triglycerides and insulin concentrations were measured with appropriate methodology and HOMA for insulin resistance (HOMA2-IR) and beta-cell function (HOMA2-B) was calculated. ${ }^{22}$ Serum hs-TnT and PlGF concentrations were measured using an electrochemiluminescence immunoassay (Roche Co., Basel, Switzerland), using the Elecsys/Cobas e411 assays. The intermediate precision Coefficient of Variation was $\leq 10 \%$ for hsTnT and 3.6-4.1\% for PIGF.

\section{STATISTICAL ANALYSIS}

Continuous variables are presented as mean \pm standard deviation (SD), or median and interquartile range in cases of skewed distributions. Categorical variables are presented as absolute (n) and relative $(\%)$ frequencies. Relations between categorical vari- ables are assessed by Fisher's exact test. Differences in means were tested by the Student t-test, or the Mann-Whitney test for small samples $(<30)$. Individual correlations between continuous variables were assessed by Pearson $r$, or Spearman rho in small samples $(<30)$.

Univariate analyses were undertaken overall (in the entire population) and stratified according to obesity (CG, Ob), and also according to MetS (CG, ObMS-, ObMS+), to identify possible significant correlates of PIGF. Finally, a linear regression model was used to incorporate and evaluate all parameters significant in the univariate analyses. Size of effect in the final regression model was estimated by Cohen's f2. Data were analyzed using Stata 11.0 statistical software (Stata Corp, Texas, USA). Based on pediatric data of PlGF, ${ }^{11}$ it was estimated that the required sample size, in order to achieve $80 \%$ power (with an alpha $=0.05$ ) in detecting PIGF differences between controls and obese children, would be 72 individuals per group. The final numbers of participants correspond to a statistical power of $48 \%$.

\section{RESULTS}

Eighty-two children were studied: 8 ObMS +, 49 ObMS- and 25 controls. Descriptive statistics are depicted in Table 1. PlGF was not related to age,

Table 1. Anthropometric and Biochemical Data at the Time of the Evaluation

\begin{tabular}{|c|c|c|c|c|}
\hline & CG $(n=25)$ & ObMS- $(n=49)$ & ObMS $+(n=8)$ & Ob (ObMS- \& ObMS,$+ n=57)$ \\
\hline Sex, females n $(\%)$ & $19(76 \%)$ & $22(44.9 \%)$ & $1(12.5 \%)$ & $23(40.4 \%)$ \\
\hline Age, years ${ }^{1}$ & $13.2 \pm 1.4$ & $11.1 \pm 1.9$ & $10.4 \pm 2.4$ & $11 \pm 2$ \\
\hline Pubertal status, in puberty, $\mathrm{n}(\%)$ & $21(84 \%)$ & $24(49 \%)$ & $3(37.5 \%)$ & $27(47.4 \%)$ \\
\hline BMI-zscore $^{1}$ & $0.67 \pm 1.11$ & $3.3 \pm 1.25$ & $3.3 \pm 1.47$ & $3.3 \pm 1.27$ \\
\hline $\mathrm{PlGF}, \mathrm{pg} / \mathrm{mL}^{1}$ & $10.9 \pm 2.8$ & $11.9 \pm 2.8$ & $14.1 \pm 3.4$ & $12.2 \pm 3$ \\
\hline HOMA2-IR ${ }^{1}$ & $2 \pm 0.9$ & $2.6 \pm 1.7$ & $2.4 \pm 1.2$ & $2.6 \pm 1.8$ \\
\hline HOMA2-B ${ }^{1}$ & $174.7 \pm 58.1$ & $199.4 \pm 69.5$ & $177.8 \pm 100.4$ & $195.9 \pm 74.6$ \\
\hline Troponin, ng/L2 & $3(3-3)^{3}$ & $3(3-3)^{4}$ & $3(3-6.9)^{5}$ & $3(3-3)^{6}$ \\
\hline Total Cholesterol, mg/dL ${ }^{1}$ & $165.4 \pm 28.2$ & $180.6 \pm 29.5$ & $180.1 \pm 49.6$ & $180.5 \pm 32.7$ \\
\hline $\mathrm{LDL}, \mathrm{mg} / \mathrm{dL}^{1}$ & $84.1 \pm 30.9$ & $114.5 \pm 27$ & $119.6 \pm 38.2$ & $115.3 \pm 28.6$ \\
\hline $\mathrm{HDL}, \mathrm{mg} / \mathrm{dL}^{1}$ & $55.6 \pm 11.4$ & $50.7 \pm 14.8$ & $36.6 \pm 5.4$ & $48.5 \pm 14.6$ \\
\hline Triglycerides, mg/dL ${ }^{1}$ & $64.9 \pm 24.5$ & $90.2 \pm 41.1$ & $119.4 \pm 52.7$ & $94.6 \pm 43.8$ \\
\hline
\end{tabular}

${ }^{1}$ mean $\pm \mathrm{SD},{ }^{2}$ median, (interquantile range),${ }^{3} 10^{\text {th }}-90^{\text {th }}$ percentile: $3.0-3.0,{ }^{4} 10^{\text {th }}-90^{\text {th }}$ percentile: $3-4.3,{ }^{5} 10^{\text {th }}-90^{\text {th }}$ percentile: $3-21.4,{ }^{6} 10^{\text {th }}-$ $90^{\text {th }}$ percentile: $3-5.3$. 
nor to BMI-z scores both overall and in all stratified analyses (all $p>0.05$ ). Puberty had no significant effect on PIGF (overall and stratified analyses, $p>0.05$ ). In the entire study population, no statistically significant differences were detected according to sex. In CG, males had significantly increased PlGF concentrations compared to females $(13.1 \pm 3.3$ vs. $10.2 \pm 2.3$, $\mathrm{p}=0.045)$. In the ObMS-, ObMS + and Ob groups, no such difference was observed.

No correlation between PIGF and HOMA2-IR or HOMA2-B was recorded (overall and stratified analyses, $\mathrm{p}>0.05$ ). Total cholesterol, LDL and triglycerides were also not related to PIGF (overall and stratified analyses, $p>0.05$ ). A weak, negative relation was noted between HDL and PIGF (Pearson's $\mathrm{r}=-0.21, \mathrm{p}=0.062$ ). Overall, PlGF was positively related to troponin (Pearson's $r=0.38, p=0.003$ ). The stratified analysis revealed that, in controls, PIGF was not related to troponin (Spearman's rho $=-0.25$, $\mathrm{p}=0.219)$. Both in ObMS- and ObMS + the correlation was positive but not significant $(\mathrm{r}=0.26$ and 0.32 , $\mathrm{p}=0.069$ and 0.428 , respectively). In the Ob group, a clear, positive relation between PLGF and troponin was documented (Spearman's rho $=0.26, \mathrm{p}=0.046$ ).

PIGF concentrations differed significantly between CG and Ob (Mann-Whitney test, $\mathrm{p}=0.048$ ). The analysis of the three groups (CG, ObMS- and ObMS +, Kruskal-Wallis test, $\mathrm{p}=0.035$ ) demonstrated that this difference occurred due to the significantly higher concentrations of PlGF in the ObMS + group compared to the CG $(p=0.009)$.

Based on the results of the univariate analysis, we ran a linear regression model including group (3 groups), troponin, HDL and sex as independent covariates. Sex and HDL were not significant factors and were subsequently removed from the model (Table 2). The outcomes were consistent with the univariate analysis, estimating that children with ObMS + had on average $2.2 \mathrm{pg} / \mathrm{mL}$ higher levels of PIGF compared to the CG. Troponin retained its significant, positive correlation with PlGF. The $\mathrm{R}^{2}$ of the final model was 0.1622 and Cohen's $\mathrm{f} 2$ was equal to 0.1936 , which lies between small (0.02) and medium (0.25) size of effect.

\section{DISCUSSION}

Our study revealed that children with the MetS have significantly increased PIGF concentrations, a marker of angiogenesis, in comparison to children without MetS. Obese children, in general, had increased PIGF concentrations compared to the nonobese, but this was mainly due to the extreme effect of the MetS subgroup. We also found higher PIGF concentrations in normal weight males than females, which was not the case in children with obesity and MetS. Lastly, we found that hs-troponin is positively correlated with PLGF in obese children.

A recent study, mentioned previously, examined PIGF concentrations in obese children ${ }^{10}$ but did not find significant differences, possibly due to the fact that only childhood obesity and not pediatric MetS was taken into account. However, in the same study, another marker of angiogenesis, VEGF, was associated with BMI z-scores in children, suggesting a potentially concomitant formation of new vessels with the expansion of adipose tissue, starting in childhood.

Published evidence in adults showed increased PIGF concentrations in patients with the MetS, ${ }^{16}$ whereas another study demonstrated PIGF expression within human atherosclerotic lesions associated

Table 2. Final regression model showing significant predictors for PIGF

\begin{tabular}{lccc}
\hline Parameter & b-coefficient $\mathbf{( 9 5 \%}$ ci) & p value & Overall F-test \\
\hline Troponin & $0.29(0.07,0.51)$ & 0.010 & p=0.003 \\
Group & & & \\
ObMS+ vs CG & $2.2(-0.1,4.5)$ & 0.061 & 0.250 \\
ObMS- vs CG & $0.8(-0.6,2.1)$ & 0.189 & $<0.001$ \\
ObMS+ vs ObMS- & $1.4(-0.7,3.6)$ & \\
Constant & $10(8.7,11.3)$ & & \\
\hline
\end{tabular}


with plaque inflammation and destabilization and clinical manifestations of the disease..$^{23}$ However, the role of PlGF in the metabolic syndrome is not totally clear. PIGF binds to the VEGF-1 receptor and forms heterodimers with free VEGF, suggesting a potential role of PIGF in modulating the actions of VEGF. ${ }^{24} \mathrm{PlGF}$ was further proposed, in a study mentioned before ${ }^{6}$ as an independent predictor of coronary heart disease after adjustment for several confounding factors, such as age, smoking, family history of myocardial infarction, physical activity and BMI, suggesting that PlGF might be involved in the formation of atherosclerotic plaques.

The association between pediatric MetS and PlGF suggests that angiogenesis may begin at an early stage of MetS development and might promote the activation of mechanisms related to the initiation of the atherosclerotic process. This is further supported by the finding that PIGF significantly correlated with hs-TnT, a biomarker related to cardiac damage, only in obese children. Indeed, the Dallas heart study ${ }^{25}$ found that in a large number of individuals, cardiac (c) TnT was undetectable among healthy subjects and that subjects with minimal cTnT elevation had underlying CVD or a high-risk phenotype for CVD. We have also previously reported in a different cohort of obese children that $\mathrm{N}$-terminal pro-brain natriuretic peptide (NT-proBNP), a direct indicator of functional and structural damage in the cardiovascular system, was increased in obese males with increased blood pressure ${ }^{26}$ and that this biomarker was associated with adiponectin levels in obese female adolescents. ${ }^{27}$ The current findings on associations between PlGF and hs-TnT, together with our previous findings on NT-proNP in a different sample of obese children, also strengthens the hypothesis that atherosclerotic risk factors are detectable or minimally elevated at a preclinical stage in children with obesity and obesityrelated clinical and metabolic comorbidities.

In our study, males with normal weight had significantly higher PlGF concentrations than normal weight females. This significant gender effect might be mediated by sex hormones, since most of the children in the control group were pubertal. This is consistent with adult studies, where PIGF concentrations are lower in pre-menopausal women than post-menopausal ones $^{28}$ in whom, in turn, PlGF concentrations were similar to those of men. Moreover, variations in PlGF concentrations have been described with the phases of the menstrual cycle and regulatory effects of the estrogens on PIGF production have been observed in pregnancy. ${ }^{28}$

The cross-sectional design of our study cannot ascertain causality in the direction of the associations. Furthermore, the instability of MetS diagnosis during adolescence is another limitation of our data. ${ }^{29}$ Carotid intima-media thickness measurements, which could contribute significantly to the understanding of our findings, were inadvertently not included in the study. ${ }^{30}$ Lastly, we solely studied PlGF concentrations as a marker of angiogenesis in children with obesity and the MetS, whereas VEGF, a more established marker of angiogenesis, was not included in the study.

Further studies are required to investigate longitudinally the associations of PIGF with the progression of MetS and atherosclerosis and to clarify whether PIGF could have a clinically significant role in the prediction of cardiovascular risk later in life.

\section{DISCLOSURES}

The authors have nothing to declare. There is no conflict of interest.

\section{REFERENCES}

1. Bridger T, 2009 Childhood obesity and cardiovascular disease. Paediatr Child Health 14: 177-182.

2. Kassi E, Pervanidou P, Kaltsas G, Chrousos G, 2011 Metabolic syndrome: definitions and controversies. BMC Med 9: 48.

3. Carmeliet P, 2003 Angiogenesis in health and disease. Nat Med 9: 653-660.

4. Sandhofer A, Tatarczyk T, Kirchmair R, et al, 2009 Are plasma VEGF and its soluble receptor sFlt-1 atherogenic risk factors? Cross-sectional data from the SAPHIR study. Atherosclerosis 206: 265-269.

5. Dewerchin M, Carmeliet P, 2012 PlGF: a multitasking cytokine with disease-restricted activity. Cold Spring Harb Perspect Med doi: 10.1101/cshperspect.a011056 (in press)

6. Cassidy A, Chiuve SE, Manson JE, Rexrode KM, Girman CJ, Rimm EB, 2009 Potential role for plasma placental growth factor in predicting coronary heart disease risk in women. Arterioscler Thromb Vasc Biol 29: 134-139.

7. Pelisek J, Well G, Reeps C, et al, 2012 Neovasculariza- 
tion and angiogenic factors in advanced human carotid artery stenosis. Circ J 76: 1274-1282.

8. Hausman GJ, Richardson RL, 2004 Adipose tissue angiogenesis. J Anim Sci 82: 925-934.

9. Makey KL, Patterson SG, Robinson J, et al, 2013 Increased plasma levels of soluble vascular endothelial growth factor receptor 1 (sFlt-1) in women by moderate exercise and increased plasma levels of vascular endothelial growth factor in overweight/obese women. Eur J Cancer Prev 22: 83-89.

10. Siervo M, Ruggiero D, Sorice R, et al, 2012 Body mass index is directly associated with biomarkers of angiogenesis and inflammation in children and adolescents. Nutrition 28: 262-266.

11. Kaess BM, Pedley A, Massaro JM, et al, 2012 Relation of vascular growth factors with CT-derived measures of body fat distribution: the Framingham Heart Study. J Clin Endocrinol Metab 97: 987-994.

12. Cullberg KB, Christiansen T, Paulsen SK, Bruun JM, Pedersen SB, Richelsen B, 2013 Effect of weight loss and exercise on angiogenic factors in the circulation and in adipose tissue in obese subjects. Obesity (Silver Spring) 21: 454-460.

13. Khurana R, Moons L, Shafi S, et al, 2005 Placental growth factor promotes atherosclerotic intimal thickening and macrophage accumulation. Circulation 111: 2828-2836.

14. Christiaens V, Lijnen HR, 2010 Angiogenesis and development of adipose tissue. Mol Cell Endocrinol 318: 2-9.

15. Lijnen HR, Christiaens V, Scroyen I, et al, 2006 Impaired adipose tissue development in mice with inactivation of placental growth factor function. Diabetes 55: 2698-2704.

16. Siervo M, Ruggiero D, Sorice R, et al, 2010 Angiogenesis and biomarkers of cardiovascular risk in adults with metabolic syndrome. J Intern Med 268: 338-347.

17. Pervanidou P, Akalestos A, Bastaki D, Apostolakou F, Papassotiriou I, Chrousos GP, 2013 Increased Circulating High Sensitivity -Troponin (hs-TnT) Concentrations in Children and Adolescents with Obesity and the Metabolic Syndrome: a Marker for Early Cardiac Damage? Metabolism 62: 527-531.

18. Cole TJ, Bellizzi MC, Flegal KM, Dietz WH, 2000 Establishing a standard definition for child overweight and obesity worldwide: International survey. BMJ 320: 1240-1243.
19. Chiotis D, Krikos X, Tsiftis G, Hatzisymeaon M, ManiatiChristidi M, Dacou-Voutetakis C, 2004 Body mass index and prevalence of obesity in subjects of Hellenic origin aged 0-18years, living in the Athens area. Ann Clin Paediatr Univ Atheniensis 51: 139-154.

20. Zimmet P, Alberti KG, Kaufman F, et al, 2007 IDF Consensus Group. The metabolic syndrome in children and adolescents - an IDF consensus report. Pediatr Diabetes 8: 299-306.

21. Tanner JM 1962 Growth at adolescence. 2nd ed. Oxford: Blackwell Scientific Publications.

22. Wallace TM, Levy JC, Matthews DR, 2004 Use and abuse of HOMA modeling. Diabetes Care 27: 1487-1495

23. Pilarczyk K, Sattler KJ, Galili O, et al, 2008 Placenta growth factor expression in human atherosclerotic carotid plaques is related to plaque destabilization. Atherosclerosis 196: 333-340.

24. Roy H, Bhardwaj S, Ylä-Herttuala S, 2006 Biology of vascular endothelial growth factors. FEBS Lett 580: 2879-2887.

25. Wallace TW, Abdullah SM, Drazner MH, et al, 2006 Prevalence and determinants of troponin T elevation in the general population. Circulation 113: 1958-1965.

26. Pervanidou P, Akalestos A, Sakka S, Kanaka-Gantenbein C, Papassotiriou I, Chrousos GP, 2010 Gender dimorphic associations between $\mathrm{N}$-terminal pro-brain natriuretic peptide, body mass index and blood pressure in children and adolescents. Horm Res Paediatr 73: 341-348.

27. Pervanidou P, Margeli A, Akalestos A, et al, 2009 Associations between circulating N-terminal pro-Brain Natriuretic Peptide (NT-proBNP) and adiponectin concentrations depend on obesity level in female adolescents: gender dimorphic findings. Horm Metab Res 41: 829-833.

28. Sorice R, Ruggiero D, Nutile T, et al, 2012 Genetic and environmental factors influencing the Placental Growth Factor (PGF) variation in two populations. PLoS One 7: e42537.

29. Goodman E, Daniels SR, Meigs JB, Dolan LM, 2007 Instability in the diagnosis of metabolic syndrome in adolescents. Circulation 115: 2316-2322.

30. Huang K, Zou CC, Yang XZ, Chen XQ, Liang L, 2010 Carotid intima-media thickness and serum endothelial marker levels in obese children with metabolic syndrome. Arch Pediatr Adolesc Med 164: 846-851. 